\title{
Systematic Approaches for Signal Processing of Soil Shaking Table Test
}

\author{
Jun Xian Lim ${ }^{1}$, Min Lee Lee ${ }^{1, *}$, and Yasuo Tanakal \\ ${ }^{1}$ Department of Civil Engineering, Lee Kong Chian Faculty of Engineering \& Science, Universiti \\ Tunku Abdul Rahman, Selangor, Malaysia
}

\begin{abstract}
Selecting an appropriate methodology in signal processing of shaking table test is of fundamental importance for evaluating the soil dynamic parameters. This paper presents the systematic approaches in selecting an appropriate signal processing method for a soil shaking table test. A soil model of $150 \mathrm{~mm}$ in diameter and $100 \mathrm{~mm}$ in height was tested on a $1 \mathrm{~g}$ shaking table. The soil model was instrumented with accelerometers to measure the resultant accelerations under different shaking motions. Under normal data processing practices, the acceleration data was adjusted and numerically integrated to obtain the linear displacement data. However, the accuracy and reliability of the computed displacement data and the adopted adjustment technique often raise arguments. In the present study, the acceleration data was subjected to two baseline correction schemes and one method of digital band-pass filtering. The results from the three signal processing methods were compared with the actual displacement data obtained from a direct measurement using laser displacement sensor. Bandpass filtering method was found to be a more reliable data processing method in this particular study. The use of laser displacement sensor can serve as a useful reference when choosing an appropriate adjustment scheme in data processing.
\end{abstract}

\section{Introduction}

Ground motion parameters and their characteristics are of importance to seismologists, geologists and earthquake engineers. Among many aspects, residual displacement is essential for investigating the fault rupture after the occurrence of a strong ground motion. The permanent or residual displacement can be caused by plastic deformation of nearsurface material or elastic deformation of ground as the result of co-seismic slip on the fault [1]. At the end of each shaking motion, the velocity should return to zero while certain amount of residual displacement can be expected. Unphysical residual displacement will be encountered if the acceleration record is not adjusted accordingly. Under common practices, the interpretation of numerically integrated displacement data from an earthquake event relies upon individual judgement, and hence exposed to numerous uncertainties. In a study of the Chi-Chi Taiwan earthquake, Boore [2] used the technology of Global Positioning System (GPS) to verify his proposed correction schemes on the residual

\footnotetext{
* Corresponding author: mllee@utar.edu.my
} 
displacement. However, the outcome of that comparison was not convincing due to large discrepancies between the computed and observed values. It may be attributed to the fact that GPS stations were not collocated with the accelerograph stations.

Over the years, various adjustment schemes for processing seismic records have been proposed by numerous authors worldwide [1- 5]. Although there are various correction schemes proposed to recover the actual shaking record, it is generally agreed that it is almost impossible to recover the earthquake record perfectly. Boore and Bommer [1] reviewed the characteristics of a strong-motion record and the effect of noises (short and long-period noise contents) on signals in an earthquake event. They also highlighted the importance of performing baseline correction and selecting a suitable cut-off frequency in digital filtering. Sensitivity analysis was carried out when examining the effect of correction schemes and other parameters on the adjusted results. Boore [2] suggested a simple baseline correction method named as zeroth-order baseline correction. This method requires removal of pre-event mean records from the entire record at the initial stage of signal processing. The subsequent procedure includes identification of obvious changes in velocity baseline. Time instant for a particular change can be identified and followed by subtracting baseline step changes in the acceleration record. After the acceleration record is baseline adjusted, it can be numerically integrated to obtain the velocity and displacement time-series. It was also concluded that the choice of baseline correction does not affect the response spectrum with periods greater than $20 \mathrm{~s}$. This finding suggested that most of the structures under seismic activities may not be affected by the choice of baseline correction method as the response spectrums are usually greater than $20 \mathrm{~s}$.

In addition, Chiu [4] suggested a "stable" three-step algorithm baseline correction scheme for processing digital strong motion data. In this method, least-square fitting and high-pass filtering of acceleration record was applied. In Japan, Ohsaki [5] proposed a wellknown correction method which is fundamentally based on the assumptions that velocity at the end of shaking will become zero whilst certain amount of residual displacement can be expected.

SeismoSignal is one of the popular commercial software to process earthquake strongmotion data. Mollova [6] presented the application of digital filtering using SeismoSignal on an actual earthquake record in Turkey. The effects of various digital filtering models (including Chebyshev, Butterworth, Bessel and Elliptic) were compared and discussed.

Besides using acceleration record to recover the displacement data in an earthquake, similar data processing technique was also applied in other engineering fields. In a study on structural behaviour of pavement, Arraigada and Partl [7] used accelerometers instead of Linear Variable Differential Transformer (LVDT) to measure the acceleration and derived the vertical deformation of pavement from the acceleration data. Slifka [8] used laser displacement sensor as a reference when processing the acceleration data of a moving object.

In light of the non-destructive nature and the ability for accurate measurement of rapidmoving object, laser displacement sensor may serve as a favourable solution for measuring small deformation in soil models. This paper focuses on signal processing of shaking table test on a soil model. Soil displacement is derived from the acceleration data obtained from accelerometers through various adjustment schemes. Laser displacement sensor is used to verify the derived displacement and serves as a reference for recommending a suitable adjustment method for the present shaking table test. 


\section{Instrumentation}

\subsection{Shaking Table System}

Figure 1 shows the shaking table setup used in the present study. A mega-torque motor was used to produce one-dimensional shaking motion on a level platform ( $2 \mathrm{~m}$ by $2 \mathrm{~m}$ ) by generating a mechanical torque repeatedly. The shaking table platform was lifted afloat by supplying an air pressure of 2 bars underneath the table platform to minimize the friction between the base and platform during cyclic horizontal movement.

The shaking table was capable of producing frequencies of $0.1-20 \mathrm{~Hz}$ and horizontal displacements of $0.5-15 \mathrm{~mm}$. Figure 2 shows the relationship between input shaking motions and the resultant peak accelerations. The highest achievable peak acceleration was about $0.55 \mathrm{~g}$.

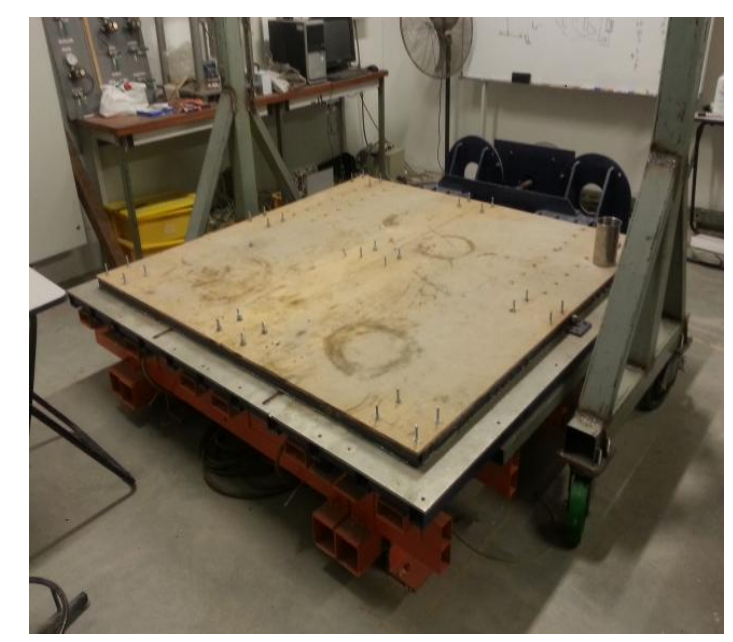

Fig. 1. Shaking table setup.

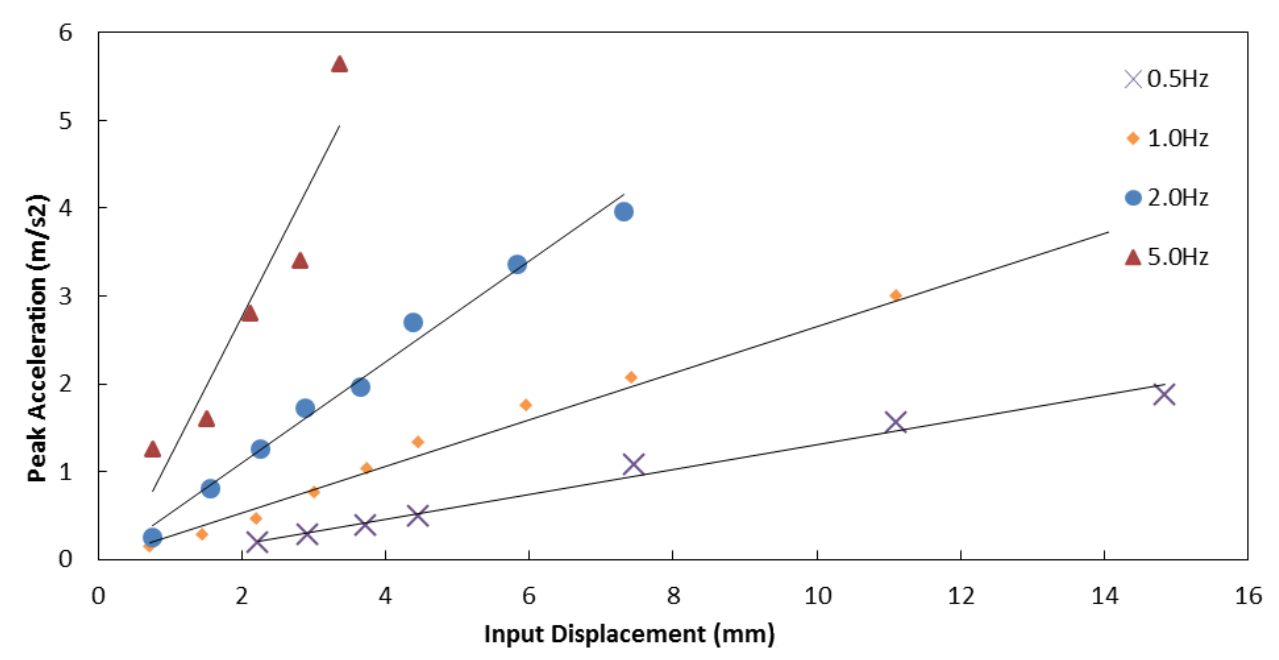

Fig. 2. Relationship between input shaking motions and resultant peak accelerations. 


\subsection{Accelerometer}

A pair of accelerometers (Tokyo Sokki Kenkyujo Co., Ltd. (TML) ARH-A model) were attached at the top and base of the soil model as shown in Figure 3. The accelerometer is a strain-gauge type transducer with a measuring range of $10 \mathrm{~m} / \mathrm{s}^{2}-500 \mathrm{~m} / \mathrm{s}^{2}$. The lowest data sampling interval is $1 \mathrm{~ms}$. The accelerometer was mounted on the top plate by using adhesive-tape. This mounting method has an advantage of providing electrical insulation between accelerometer and the contact surface [9].

\subsection{Laser Displacement Sensor}

A laser displacement sensor (Optex CD5 model) was installed near the base of the soil model to provide a direct displacement measurement (Figure 4). The sensor has a measuring range of $85 \pm 20 \mathrm{~mm}$, with a resolution of $1 \mu \mathrm{m}$ and a sampling interval as low as $100 \mu \mathrm{s}$.

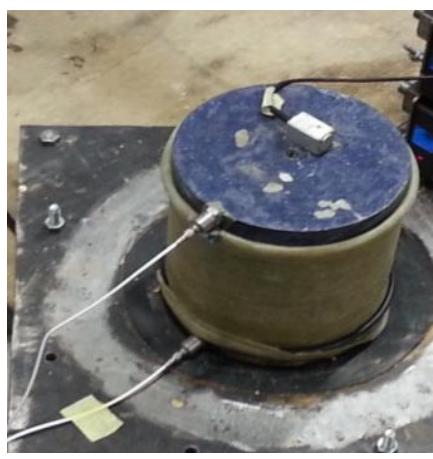

Fig. 3. Soil model and accelerometers.

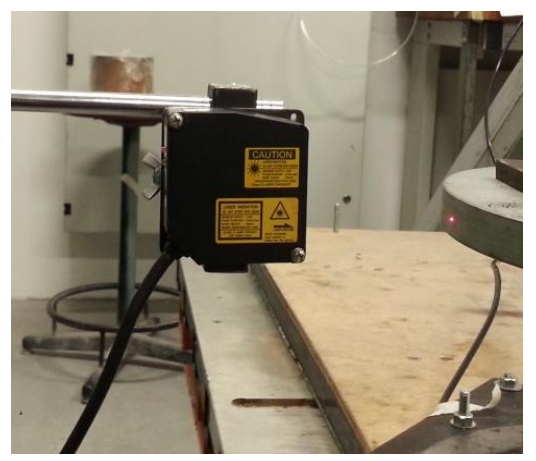

Fig. 4. Laser displacement sensor.

\section{Sample Preparation}

A remoulded soil sample was prepared for the shaking table test (Figure. 3). Based on the British Soil Classification System, the soil can be classified as Sandy Silt. The soil was compacted to $95 \%$ of the maximum dry density $\left(1664 \mathrm{~kg} / \mathrm{m}^{3}\right)$. The nominal diameter of the sample was $150 \mathrm{~mm}$ while the height was $100 \mathrm{~mm}$. The soil sample was confined by a prefabricated rubber membrane and then tightened by a pair of O-ring at top and bottom of the sample. Air confining pressure was supplied into the cylinder chamber and allowed to stabilize for 15 mins.

\section{Data Acquisition, Processing \& Analysis}

Figure 5 shows typical displacement waveforms measured by the laser displacement sensor with shaking motions of $2 \mathrm{~Hz}, 4 \mathrm{~Hz}$ and $6 \mathrm{~Hz}$. The shaking table setup was capable of producing a fairly consistent and smooth cyclic movement at different frequencies. 

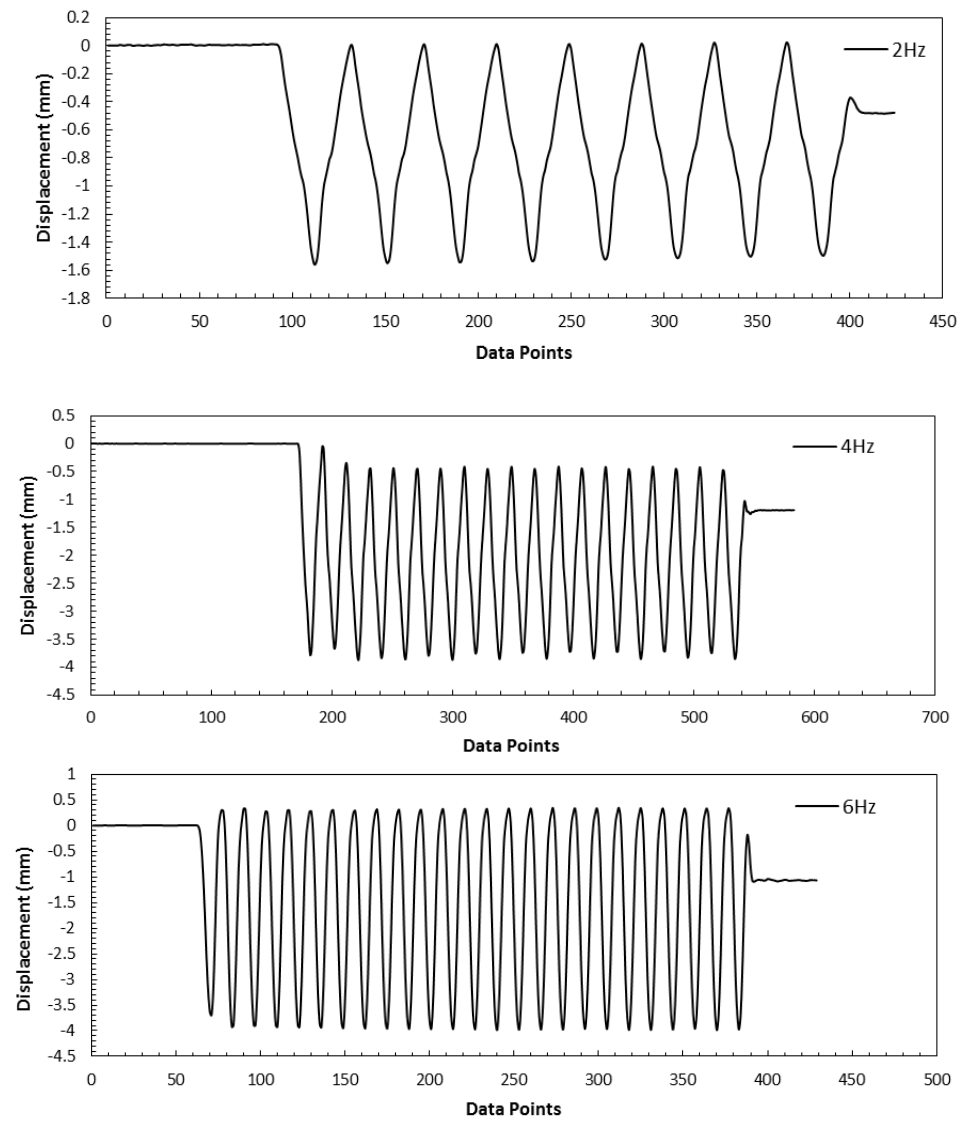

Fig. 5. Displacement waveforms measured using laser displacement sensor.

Figure 6 shows the typical raw acceleration time-series plots obtained from accelerometer measurements. Apparently, an appropriate signal processing approach was required to eliminate the undesirable background noises associated with the acceleration data. 

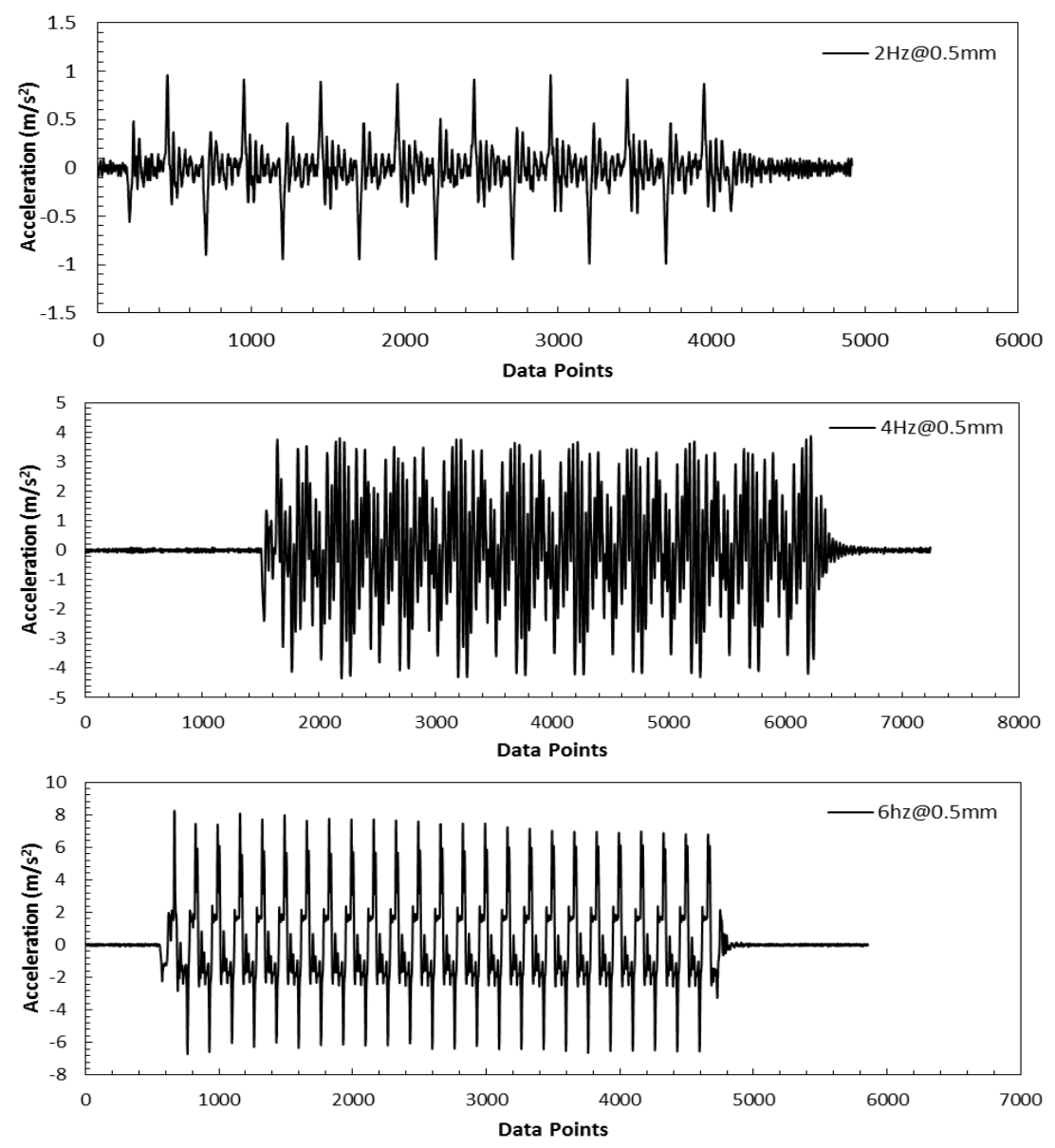

Fig. 6. Acceleration records from accelerometer under different shaking frequencies.

Velocity and displacement time-series can be obtained through numerical integration of the linear-approximated acceleration data points and the assumed initial conditions (i.e. initial velocity and initial displacement). The detailed procedures of the numerical integration may refer to $[5,10]$.

There is a significant baseline shift in the velocity and displacement profiles, as shown in Figures 7 and 8 . The displacement waveform gradually shifted towards the negative sign owing to the negative DC bias in the acceleration time-series record.

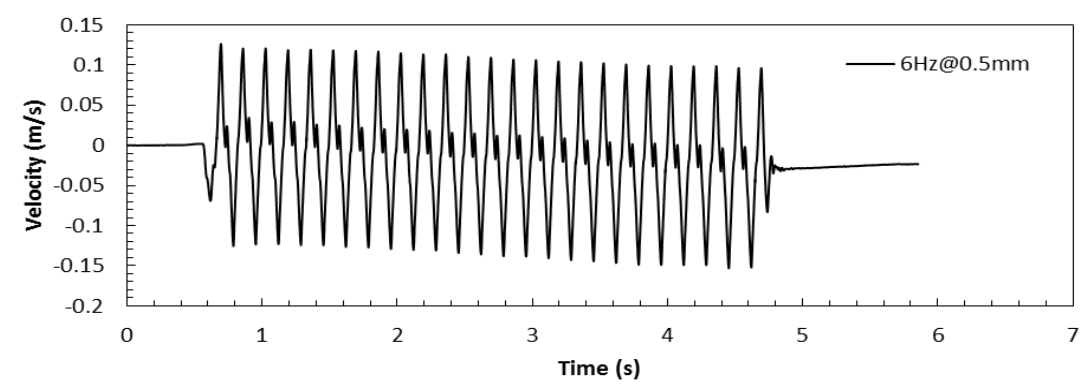

Fig. 7. Derived velocity time-series plot through numerical integration. 
If no correction were to be done, the residual displacement at the end of shaking would be unreasonable. As shown in Figure 8, the residual displacement is about $9 \mathrm{~cm}$ which was far greater than the input and actual displacement. Therefore, the original shaking record has to be corrected so as to avoid the wavy nature of the displacement waveform. The baseline shift may be attributed to many reasons including ground tilting [11], and contamination of low-frequency noise. Baseline correction is found to be effective in removing low-frequency content and it is actually a method of high-pass filtering with an unknown cut-off frequency [1].

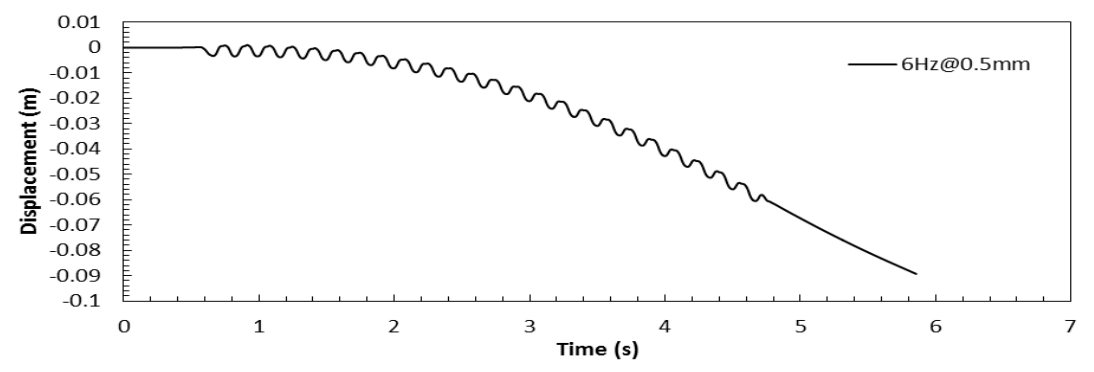

Fig. 8. Derived displacement time-series plot through numerical integration.

The original acceleration record was corrected by attempting three well-established correction schemes, namely Ohsaki's baseline correction, simple quadratic baseline correction, and bandpass filtering methods. The corrected outputs in comparison with the displacement data obtained from the direct laser displacement measurement are shown in Figure 9. It should be noted that the computed displacement traces from accelerometer and the measurement from laser displacement sensor were not oscillated about the same baseline. This is attributed to two standalone datalogger used when capturing the data. The computation of baseline correction by Ohsaki [5] was performed with the aids of a coded programming language, FORTRAN. Apparently, the Ohsaki's baseline correction method renders a movement with a significant residual displacement at the end of shaking. This result was inconsistent with the measurements from the laser displacement sensor (Figure 9).

Through simple quadratic baseline correction method, the problem of baseline drift was remediated significantly as compared to the Ohsaki's baseline correction method. The simple quadratic approach involves subtracting the entire acceleration record from a quadratic least-square fitting line prior to numerical integration. This approach is widely used in commercial processing programs, e.g. SeismoSignal.

Since the baseline-corrected record shows somewhat wavy nature of waveform, it is believed that high-pass or band-pass filtering is capable of removing the low-frequency unwanted signal. Boore and Bommer [1] suggested an approach for selecting a reasonable cut-off frequency in low-pass filtering. In a Fourier Amplitude Spectrum (FAS) plot, shaking record was compared with pre-event or post-event record. Higher cut-off frequency was decided at a frequency where the record to noise ratio is below three. Besides, the frequency response of accelerometer, i.e. $50 \mathrm{~Hz}$ should be considered when selecting an appropriate cut-off frequency. The relevant comparison is depicted in Figure 10. Nevertheless, the process in choosing a corner frequency in the present study requires a trial-and-error effort.

Band-pass 4th-order Butterworth filtering was subsequently attempted in this study. The filtered results were then compared to that of the laser displacement sensor. Phase difference between the two results can still be observed owing to the filtering process. It was decided to set the amplitude difference between the displacements obtained from 
filtering method and laser sensor measurement to within $10 \%$, as a criterion when choosing a suitable correction scheme. Slifka [8] reported a similar criterion except that their study involved two types of errors: standard error and peak error. From Figure 9, the displacement waveform computed from the bandpass filtering method does not show any noise effect caused by the low and high-frequency noises. Since the results from bandpass filtering method provided a signal that fulfilled the above-mentioned criterion, it was regarded as the most suitable data processing method in this particular study.

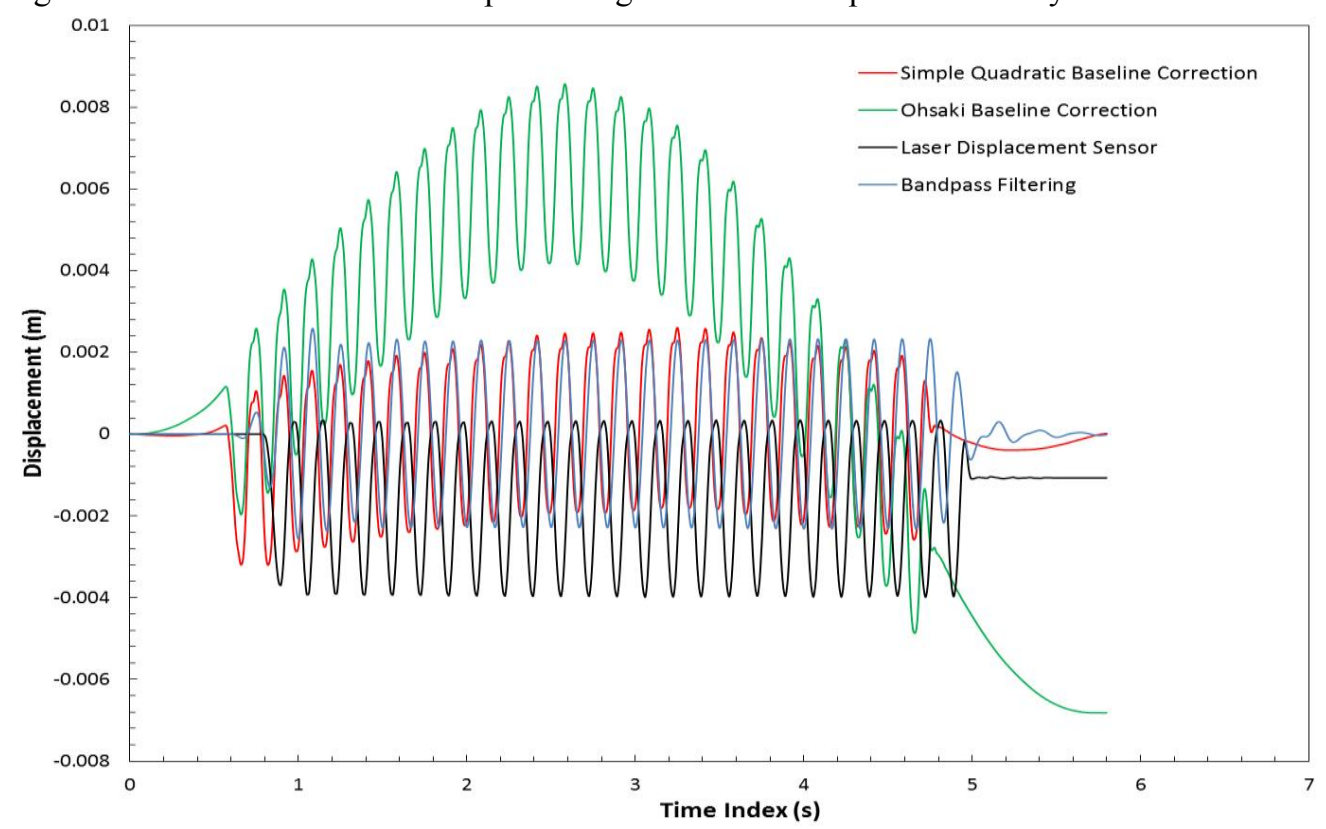

Fig. 9. Comparisons of baseline correction and bandpass filtering methods with laser displacement measurement (6Hz@0.5mm).

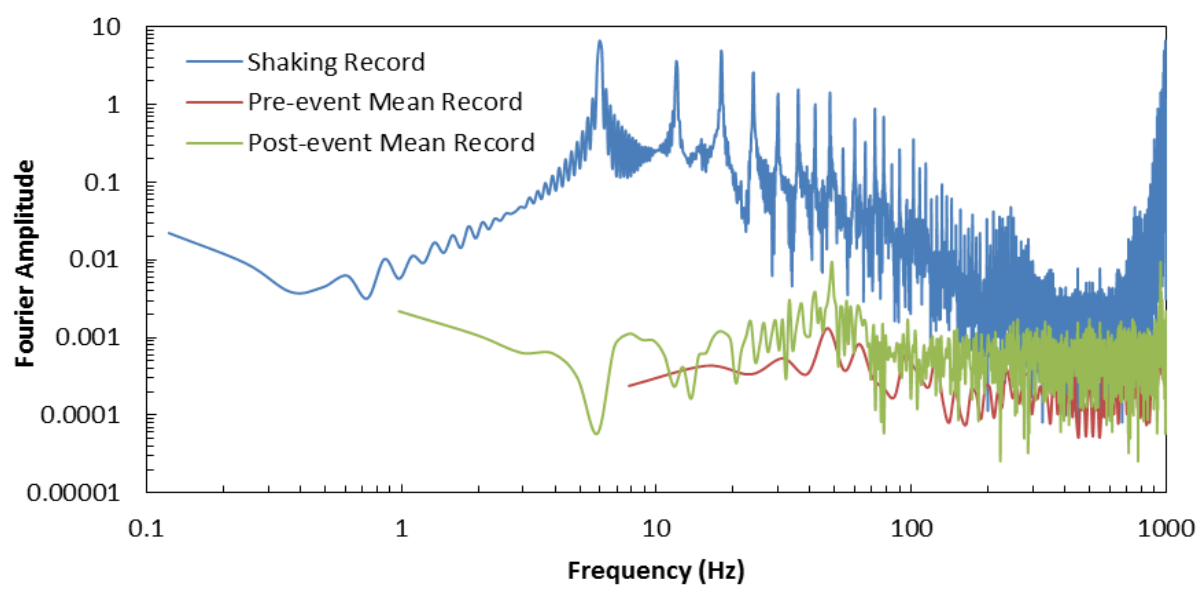

Fig. 10. Comparison of Fourier Amplitude Spectrum for shaking record, pre-event \& post-event mean records (6Hz@0.5mm).

Figure 11 shows a typical hysteresis loop obtained upon processing through the bandpass filtering. From the hysteresis loop, secant shear modulus and damping ratio can 
be easily determined by established methods. Despite of the advantage of using laser displacement sensor as highlighted in the present study, the functions of accelerometer in soil dynamic study should not be overlooked. In many circumstances, acceleration transducer is still required for the measurement of ground response to obtain the synchronized acceleration, velocity and displacement data [12-15].

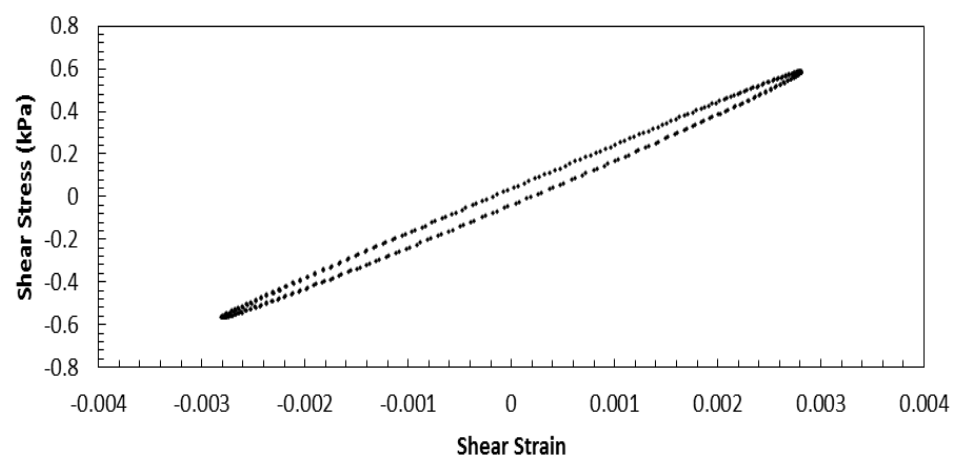

Fig. 11. Typical hysteresis loop for the tested soil model.

\section{Conclusion}

This study reports the data processing procedures of a shaking table test on a small scaled soil model. Followings are the conclusions that can be drawn from the present study:

(1) A systematic approach of signal processing for a soil shaking table test should include a reliable reference for benchmarking the processed data. Laser displacement sensor can be used as a reliable reference (intuitively similar to the role of a GPS in seismology) when recommending an appropriate adjustment scheme in data processing.

(2) Numerous data processing / filtering methods should be attempted in order to choose the most suitable approach for a particular study.

(3) Digital band-pass filtering is found to be the most suitable method in this particular study. The integrated displacement profile matches reasonably well with the laser displacement measurement.

\section{Acknowledgment}

The authors would like to acknowledge the financial supports from the Fundamental Research Grant Scheme (FRGS), Malaysia (Grant No.FRGS/2/2014/TK02/UTAR/02/1).

\section{References}

1. D.M. Boore, J.J. Bommer, SOIL DYN EARTHQ ENG., 25, 93-115 (2005)

2. D.M. Boore, B SEISMOL SOC AM., 92, 1199-1211 (2001)

3. W.D. Iwan, M.A. Moser, C.Y. Peng, B SEISMOL SOC AM., 75, 1225-1246 (1985)

4. H.C. Chiu, B SEISMOL SOC AM., 87, 932-944 (1997)

5. Ohsaki. Y, Introduction to Spectrum Analysis of Earthquake Motion (Kajima Shuppankai, 1994) 
6. G. Mollova, EURASIP-JASP., 2007, 1-9 (2006)

7. M. Arraigada, M. Partl, 6th Swiss Transport Research Conference (2006)

8. L.D. Slifka, An Accelerometer Based Approach to Measuring Displacement of a Vehicle Body (Msc Thesis, University of Michigan, 2004)

9. A. Piersol, T. Paez, Harris' Shock and Vibration Handbook (Mc GrawHill Education, 2009)

10. G.V. Berg, G.W. Housner, B SEISMOL SOC AM., 51, 175-189 (1961)

11. V. Graizer, B SEISMOL SOC AM., 96, 2090-2102 (2006)

12. Kazama. M, Yanagisawa. E, Eleventh World Conference on Earthquake Engineering, 367 (1996)

13. Kazama. M, Toyota. H, Towhata. I, Yanagisawa. EJGE., 535/III(34), 73-82 (1996)

14. Koga. Y, Matsuo. O, SOILS FOUND., 30, 162-174 (1990)

15. A.J. Brennan, N.I. Thusyanthan, S.P.G. Madabhushi, J GEOTECH GEOENVIRON., 131, 1488-1497 (2005) 